PAPER

\title{
Improvement of sound insulation of doors or windows by absorption treatment inside the peripheral gaps
}

\author{
Takumi Asakura $^{1, *}$ and Shinichi Sakamoto ${ }^{2}$ \\ ${ }^{1}$ Institute of Technology, SHIMIZU Corporation, \\ 3-4-17 Etchujima, Koto-ku, Tokyo, 135-8530 Japan \\ ${ }^{2}$ Institute of Industrial Science, the University of Tokyo, \\ 4-6-1 Komaba, Meguro-ku, Tokyo, 153-8505 Japan
}

(Received 29 May 2012, Accepted for publication 16 November 2012)

\begin{abstract}
Slit-shaped gaps included in building elements such as doors or windows are apt to deteriorate the sound insulation performance of the wall system. In order to reduce sound energy transmitted through these parts, one of the practical measures is to place sound absorption materials inside the gaps. In this study, the effect of such an absorption treatment was examined through numerical and experimental studies. In the numerical study, the effect of sound absorption treatment inside the gaps existing around doors/windows was examined by applying the finite-difference timedomain (FDTD) method. To examine the results of the numerical study, a full-scale model experiment was performed with the sound intensity measurement method. In addition, a case study was performed to confirm the effect of absorption treatment of the peripheral gap of a door set in a real office space, and the effect was also confirmed by numerical study and measurement. As a result, it was found that sound insulation degradation caused by sound transmission through the gaps can be improved by sound absorption treatment inside the gaps.
\end{abstract}

Keywords: Leak transmission, Absorption treatment, Gap, Finite-difference time-domain method

PACS number: 43.55.Rg, 43.55.Dt [doi:10.1250/ast.34.241]

\section{INTRODUCTION}

To maintain a comfortable sound environment inside rooms, the sound insulation performance of the architecture is one of the most important factors. In particular, in a modern city, residential buildings are located at sites neighboring roadways with a large traffic volume, and prevention of the leakage of such urban noise into residential spaces is necessary. In such a situation, the building elements of windows or doors have relatively low sound insulation performance and these elements can be a bottleneck in sound insulation. For this reason, improvement of the sound insulation of such building elements is necessary.

One of the most critical factors affecting the sound insulation across building elements is sound transmission through narrow gaps that occur in the peripheral parts of movable building elements, such as windows and doors. The sound leakage through such gaps deteriorates the sound insulation performance of the elements. The effect of

*e-mail: t_asakura@shimz.co.jp sound transmission through the gaps or apertures has been theoretically investigated [1-5]. More practical case studies on the sound insulation performance of real building elements such as doors and windows have also been carried out [6-10]. Lewis [6] and Burgess [7] investigated the sound insulation of windows focusing on the sound transmission through the frame construction of the windows. Hongisto and coworkers investigated a method of predicting the sound insulation of the door construction, considering the sound transmission through the door panel and the peripheral gaps [8,9]. Iwase et al. performed an experimental investigation on various characteristics of sound leakage from a window sash using a particle velocity sensor [10]. While research on sound transmission through gaps is plentiful, as described above, no investigations on prevention of sound leakage through such peripheral gaps has been reported.

In our past study, we investigated the improvement of the sound insulation characteristics of a ventilation opening with a duct shape [11]. In that study, a series of Helmholtz resonators were designed and installed inside the duct, and the sound insulation characteristics were considerably 
improved. In order to improve the sound insulation of slits or apertures, a scheme in which reactive-type sound insulation devices are included inside the gaps is applicable. However, a reactive-type device often requires a relatively large volume, and it is hard to set such devices inside gaps that have little interior air volume. On the other hand, as a space-saving scheme for sound insulation, an absorption treatment in which porous materials are introduced into the gaps has the possibility to improve the sound insulation.

In this study, therefore, we investigated the absorption effect of porous materials inside narrow gaps with several different cross sections by performing a full-scale model experiment and numerical analysis. In order to analyze the absorption effects of the porous material, we apply the finite-difference time-domain (FDTD) method. This method is one of the wave-based numerical methods, such as FEM and BEM, and has been widely used in the field of acoustics. In the FDTD calculation, a numerical scheme [12] that is able to simulate sound propagation inside a porous material on the basis of the theory of the Rayleigh model is applied. In addition to the basic study, in situ measurement of the sound insulation performance of a door panel in an office space was performed with and without absorption treatment of the peripheral gaps, and the acoustical effects of the absorption treatment are discussed.

\section{SOUND TRANSMISSION CHARACTERISTICS OF NARROW GAPS WITH ABSORPTION TREATMENT}

In order to examine the sound transmission characteristics of narrow gaps with absorption treatment, gaps with several different cross sections, as shown in Fig. 1, were investigated. These cross sections represent typical models of gaps existing in the peripheral parts of doors and windows. Firstly, absorption treatment inside straight gaps was investigated. In this case, gaps with one-/both-side absorption were investigated. This gap is called Type A, and 12 conditions were investigated by changing the placement of the sound absorption layer (one side and both sides), its thickness $(15 \mathrm{~mm}, 30 \mathrm{~mm}$, and $45 \mathrm{~mm}$ ), and the density of the sound absorbing material (glass wool: $32 \mathrm{~kg} / \mathrm{m}^{3}$ and $96 \mathrm{~kg} / \mathrm{m}^{3}$ ). Secondly, bent gaps were investigated. The basic type of these gaps, Type F1, had $4 \mathrm{~mm}$ width. The middle part of the gap was varied to investigate two conditions: $30 \mathrm{~mm}$ wide with $96 \mathrm{~kg} / \mathrm{m}^{3}$ glass wool (Type F2) and $70 \mathrm{~mm}$ wide with the same material (Type F3).

Sound insulation characteristics of these gaps were investigated by numerical simulation and laboratory experiment. Details of the method are described hereafter. (a) Straight gap

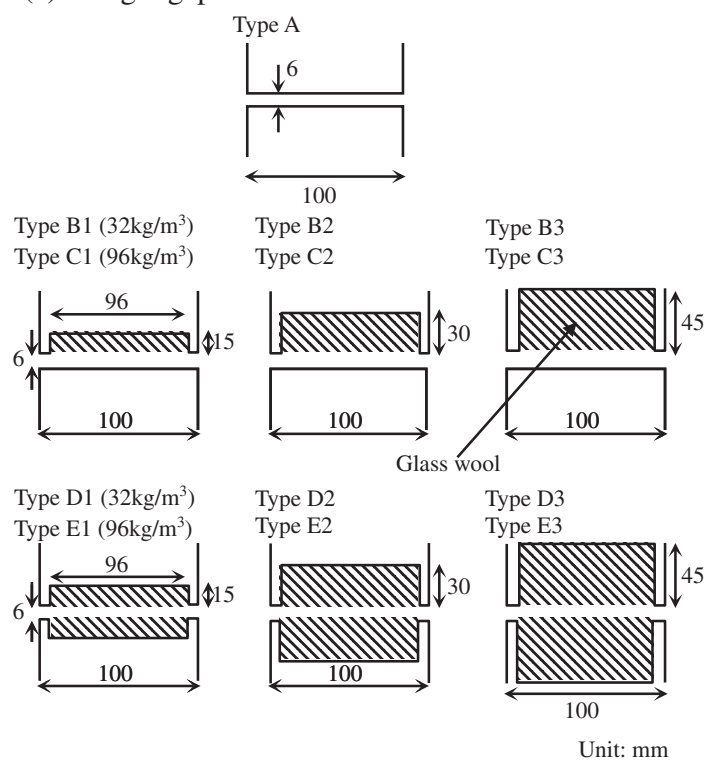

(b) Bent gap
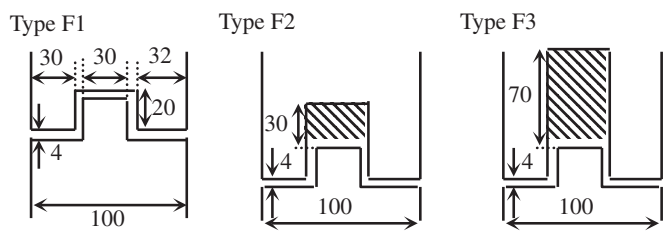

Unit: mm

Fig. 1 Cross sections of narrow gaps with straight/bent gaps.

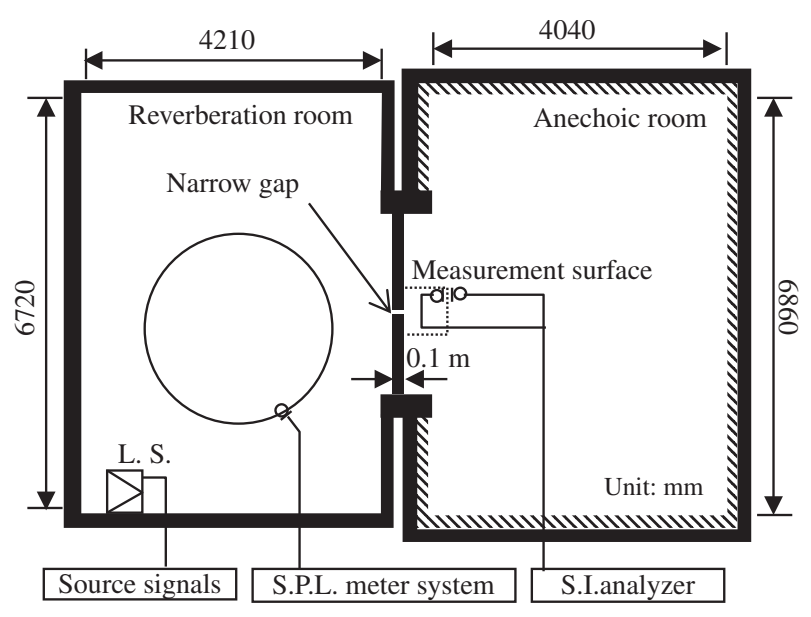

Fig. 2 Setup of the laboratory experiment.

\subsection{Laboratory Experiment}

Figures 2 and 3 show the experimental setup for the measurement of sound transmission loss, and detailed dimensions of the slit-shaped gaps and the measurement surface, respectively. A gap $100 \mathrm{~mm}$ wide and $1,800 \mathrm{~mm}$ long was made in the separation wall, as shown in Fig. 3. The measurement was performed by the scanning intensity method referring to ISO 15186-1 [13]. The measurement surface, whose axonometric figure and cross section are shown in Fig. 3, is set inside an anechoic room, and the 
(a) Axonometric figure

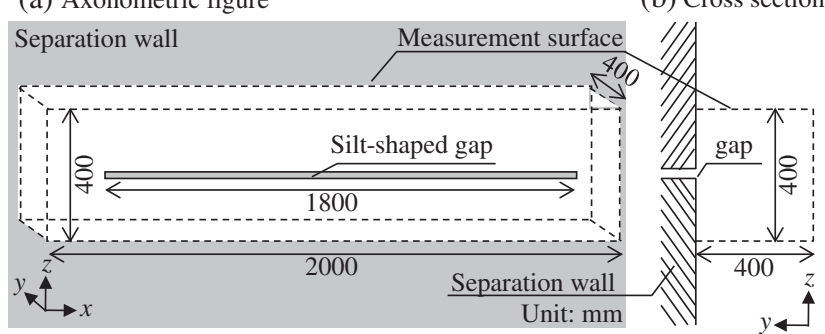

Fig. 3 Axonometric figure of the slit-shaped gap and the measurement surface for intensity measurement.

sound intensities on the surface were measured by the twomicrophone method. Then, the sound energy transmitted through the gap was obtained by integrating the sound intensities on the surface. Sound transmission loss $R$ was calculated by

$$
R=\bar{L}_{p}-6-\left(\bar{L}_{\mathrm{In}}+10 \log _{10}\left(\frac{S_{\mathrm{m}}}{S_{\mathrm{gap}}}\right)\right),
$$

where $\bar{L}_{p}$ is the average sound pressure level in the source room, $\bar{L}_{I \mathrm{n}}$ is the average normal sound intensity level on the measurement surface set in the receiving room, $S_{\mathrm{m}}$ is the total surface area of the measurement surface, and $S_{\text {gap }}$ is the projected area of the gap opening.

\subsection{Numerical Analysis}

The sound transmission loss through the gap was calculated by the two-dimensional FDTD method. The propagation of sound waves inside absorption material is included in the FDTD calculation with an assumed flow resistance of the material. The numerical scheme is described hereafter.

The sound wave in a two-dimensional sound field is described by the following equations. Equations (2) and (3) are the momentum equations in the $x$ - and $y$-directions, respectively, and Eq. (4) is the continuity equation.

$$
\begin{aligned}
& \frac{\partial p}{\partial x}+\rho \frac{\partial u_{x}}{\partial t}=0 \\
& \frac{\partial p}{\partial y}+\rho \frac{\partial u_{y}}{\partial t}=0 \\
& \frac{\partial p}{\partial t}+\rho c^{2}\left(\frac{\partial u_{x}}{\partial x}+\frac{\partial u_{y}}{\partial y}\right)=0
\end{aligned}
$$

Here, $p$ is the sound pressure, $u_{x}$ and $u_{y}$ are the particle velocities in the $x$ - and $y$-directions, respectively, $\rho$ is the density of the air, and $c$ is the speed of sound in air.

On the other hand, sound propagation inside a porous material with an isotropic property is described as follows, on the basis of the Rayleigh model [14].

$$
\frac{\partial p}{\partial x}+\rho_{\mathrm{e}} \frac{\partial u_{x}}{\partial t}+R \cdot u_{x}=0
$$

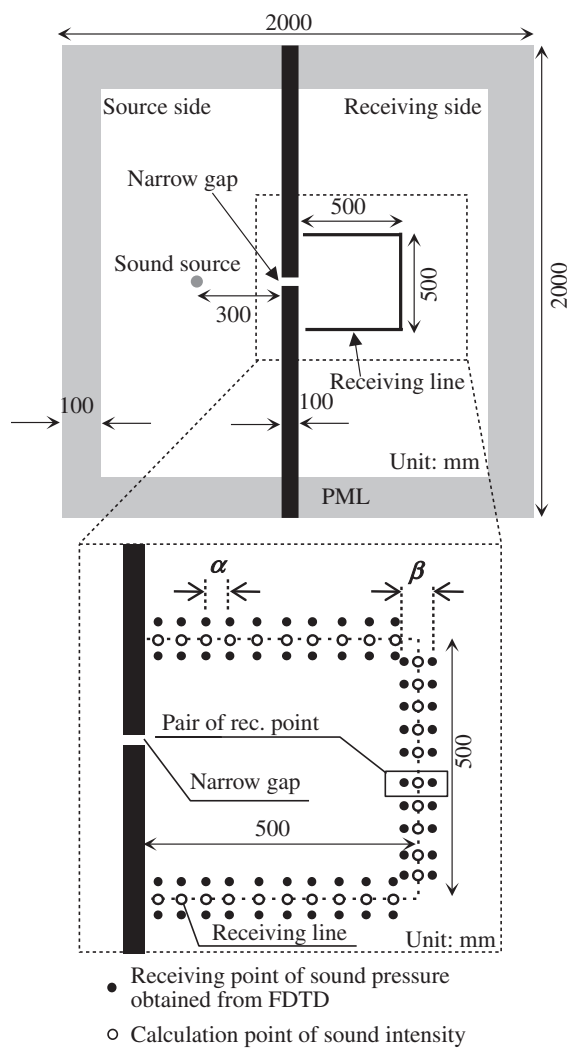

Fig. 4 Two-dimensional sound field for calculating the sound transmitted through the gap.

$$
\begin{aligned}
& \frac{\partial p}{\partial y}+\rho_{\mathrm{e}} \frac{\partial u_{y}}{\partial t}+R \cdot u_{y}=0 \\
& \frac{\partial p}{\partial t}+\frac{\rho_{\mathrm{e}} c_{\mathrm{e}}^{2}}{\Omega}\left(\frac{\partial u_{x}}{\partial x}+\frac{\partial u_{y}}{\partial y}\right)=0
\end{aligned}
$$

Here, $\Omega$ is the porosity, $c_{\mathrm{e}}$ is the speed of sound inside the porous material, $R$ is the flow resistance, and $\rho_{\mathrm{e}}$ is the effective density of the material. The spatial and time derivatives in the described equations can be approximated by central finite difference forms, and they are transformed to equations in discretized forms. Details of the discretized equations are described elsewhere [15]. Using the equations, the time development of sound propagation inside the air/porous material is calculated.

Figure 4 shows the two-dimensional sound field set for the FDTD calculation. To assume a nonreflection boundary condition, the sound field is terminated with a perfectly matched layer (PML) [16]. A narrow gap is set inside the separation wall between the source and receiving sides. As shown in the figure, the sound source in which the initial sound pressure distribution has a Gaussian profile that includes frequency components up to $10 \mathrm{kHz}$ is set in front of the gap. The frequency components of the calculated impulse responses ranged from $89.0 \mathrm{~Hz}$ to $5.61 \mathrm{kHz}$, which includes $1 / 3$ Oct. bands from $100 \mathrm{~Hz}$ to $5 \mathrm{kHz}$. In this 


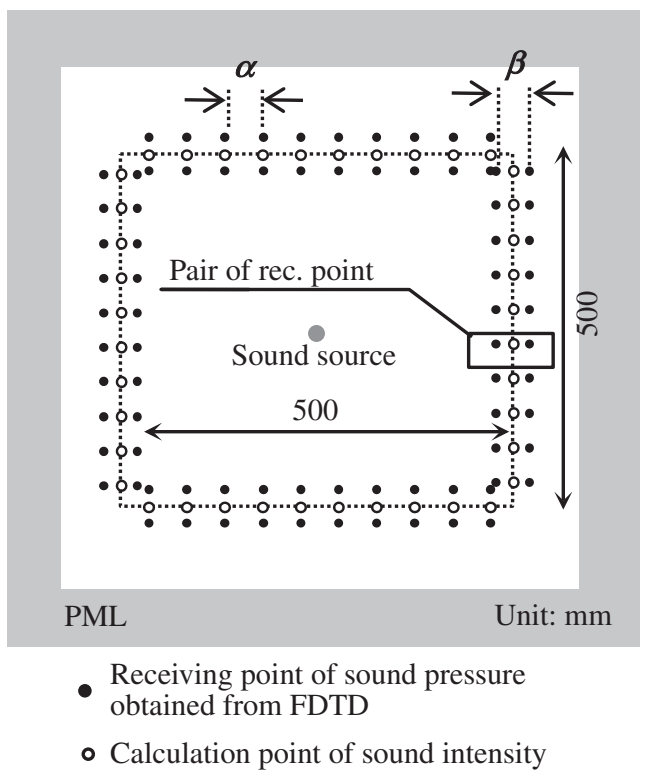

Fig. 5 Two-dimensional sound field for calculating the sound energy level of the sound source.

calculation, the normal incidence transmission loss $R_{0}$ is calculated by

$$
R_{0}=L_{J, \mathrm{i}}-L_{J, \mathrm{t}},
$$

where $L_{J, \mathrm{i}}$ is the sound energy level of the incident sound and $L_{J, t}$ is that of the sound transmitted through the gap. These sound energy levels were calculated following the theory of cross-spectrum method [17]. In this calculation, multiple receiving points, as indicated by black circles in Fig. 4, are set along the closed receiving line, and the transient responses at the points are obtained. Then, the sound intensities defined on the closed receiving line, as indicated by whitened circles, are calculated by applying the cross-spectrum method to every pair of transient responses surrounded by the broken line. Details of the calculation algorithm are described in [17]. Then, all the sound intensities are integrated, and the sound energy level $L_{J, \mathrm{t}}$ is obtained. The distances $\alpha$ and $\beta$ (as shown in Fig. 4) were both set to be $50 \mathrm{~mm}$. Overall, transient responses at 60 receiving points were used in the calculation.

On the other hand, the sound energy incident to the aperture $L_{J, \mathrm{i}}$ is calculated from the sound energy of the sound source. The sound energy of the sound source is calculated using the results of another FDTD calculation for the sound field shown in Fig. 5. The sound energy of the sound source is obtained by spatial integration of the sound intensities on the closed line shown in Fig. 5. The calculation method for the sound intensities is the same as that described above. In this calculation, the distances, $\alpha$ and $\beta$ were both set to be $50 \mathrm{~mm}$ and transient responses at 80 receiving points were used.
In the FDTD calculation, a discrete spatial grid size of $2.0 \times 10^{-3} \mathrm{~m}$ and a time interval of $4.0 \times 10^{-3} \mathrm{~ms}$ were chosen. The normal incidence absorption coefficients of surfaces inside the gap were set to be 0.02 by adopting a normal acoustic impedance of $62,000 \mathrm{Ns} / \mathrm{m}^{3}$. Flow resistances of $10,000 \mathrm{Ns} / \mathrm{m}^{4}$ and $50,000 \mathrm{Ns} / \mathrm{m}^{4}$ were set for glass wool of $32 \mathrm{~kg} / \mathrm{m}^{3}$ and $96 \mathrm{~kg} / \mathrm{m}^{3}$ density [18], respectively, and the porosity was assumed to be 1.0. The speed of sound and the density inside the material were set to be the same as those in air for simplicity.

\subsection{Results and Discussion}

\subsubsection{Straight gaps}

Figures 6 and 7 show the calculation and measurement results, respectively, for straight gaps with and without absorption treatment. In these figures, arrows indicate the dip frequency bands in which the lowest sound transmission loss appeared.

In the calculation results, tendencies of the frequency characteristics described below are seen. The first is that the higher the frequency bands are, the higher the transmission loss becomes in the case with absorption. The second is that the thicker the absorption material becomes, the higher the sound insulation performance becomes. The third is that the thicker the absorption material becomes, the lower the dip frequency bands become, as the arrows indicate in the figure, and as a result of this phenomenon, the sound insulation in the lower range is progressively deteriorated by increasing the volume of glass wool. In addition to these findings, gaps with glass wool of $96 \mathrm{~kg} / \mathrm{m}^{3}$ density are found to have higher transmission loss, in the middle- and high-frequency ranges, than gaps with glass wool of $32 \mathrm{~kg} / \mathrm{m}^{3}$ density. Likewise, gaps with both-side absorption treatment have higher transmission loss than the gaps with only one-side absorption treatment.

In the measurement results shown in Fig. 7, the same tendencies as those in the calculation results, from the viewpoint of the improved characteristics of sound insulation in the middle- and high-frequency ranges, are seen, although a slight disagreement in the dip frequency bands between the measurement and the calculation is evident.

\subsubsection{Bent gaps}

Figures 8 and 9 show the calculation and measurement results, respectively, of the bent gaps with and without absorption treatment.

In the calculation results, by comparing Type F1 with Types F2 and F3, we see that the sound transmission loss in the frequency range from $630 \mathrm{~Hz}$ to $2.5 \mathrm{kHz}$ increases when setting the absorption material inside the gap. However, as the volume of the space for setting the material is larger in Type F3 compared with Type F2, the dip frequency indicated by arrows decreases from the 


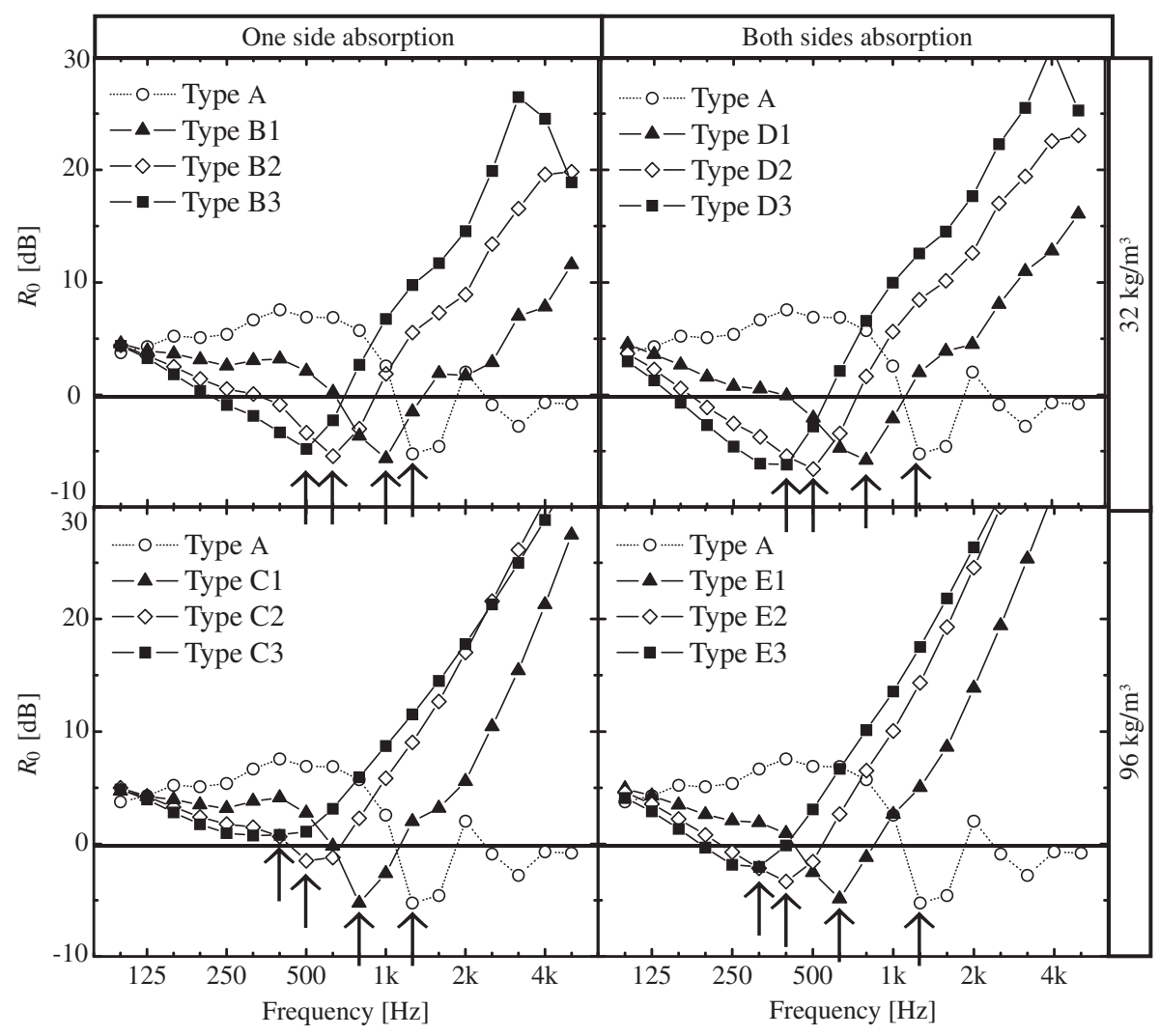

Fig. 6 Calculation results of the straight gaps with and without absorption treatment.

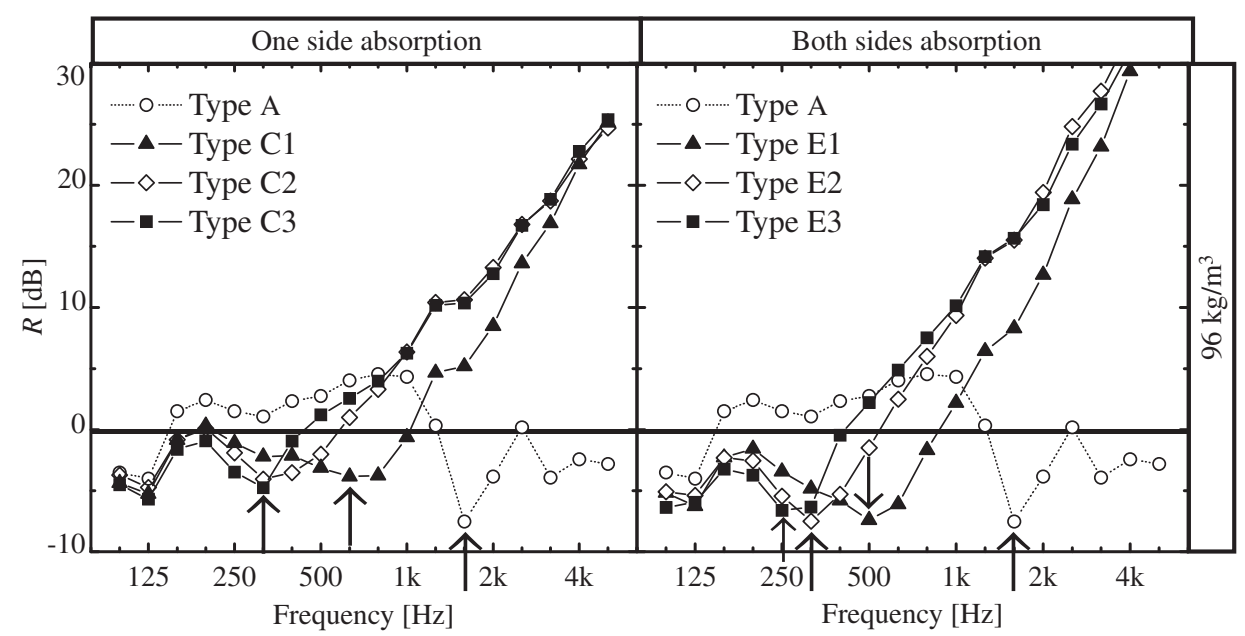

Fig. 7 Measurement results of the straight gaps with and without absorption treatment.

$630 \mathrm{~Hz}$ band to the $315 \mathrm{~Hz}$ band, and the sound insulation performance is deteriorated as a result of the shift in the dip frequency.

In the measurement results shown in Fig. 9, the same characteristics as those described above are seen, whereas the tendency of the frequency characteristics in the lower frequency range shows a slightly different feature from that of the calculation.

\subsubsection{Consideration of absorption characteristics}

In the results of straight/bent gaps, the characteristics described below were common. The sound insulation performance was improved in the middle- or highfrequency range by inserting absorption materials into the gaps. However, at some frequency bands in the lower range, the sound insulation performance was deteriorated as a result of the shift of the critical dip frequency bands 


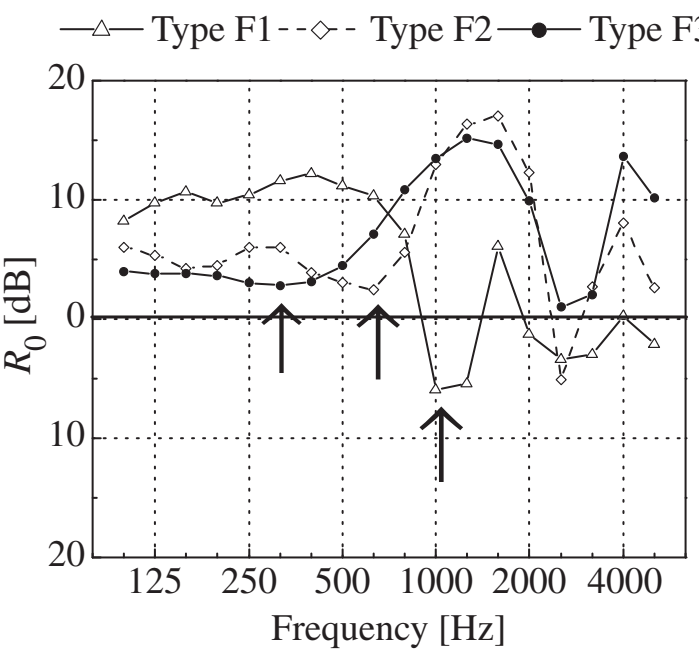

Fig. 8 Calculation results of the bent gaps with and without absorption treatment.

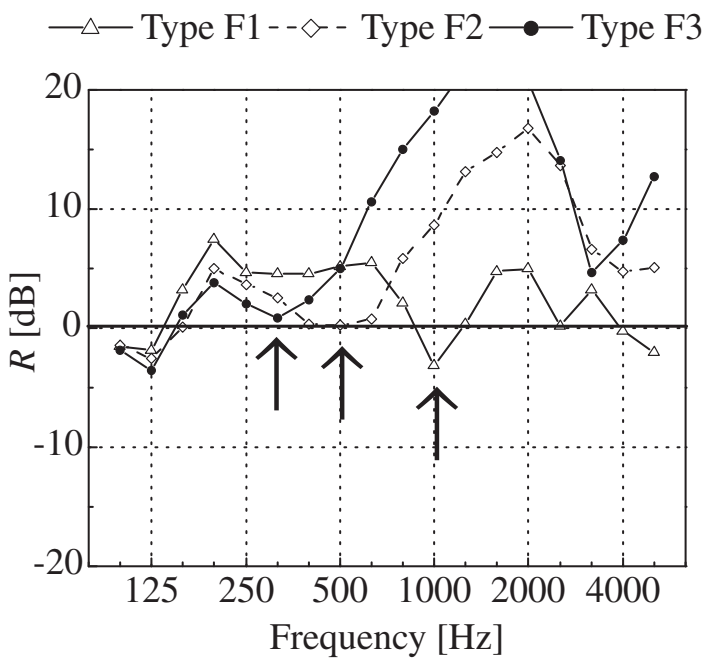

Fig. 9 Measurement results of the bent gaps with and without absorption treatment.

to lower ranges, which was caused by the increased volume/thickness of the absorption layer inside the gaps. In order to investigate the mechanism behind this phenomenon, the transmission losses of bent gaps without absorption treatment, Types $\mathrm{F}^{\prime}$ and $\mathrm{F}^{\prime}$ as shown in Fig. 10, were additionally measured. The results are shown in Fig. 11. The decrease in the dip frequencies is also seen for these types of gaps without absorption materials. This result reveals that the shift of the dip frequency is caused by setting an air space along the gap path.

The acoustical effect caused by the air volume inside the gaps was investigated by the transfer matrix method. By treating the air space as a muffler device, the reactive effect of this system was calculated. Details of this calculation method are described in the appendix. Types $\mathrm{F} 1, \mathrm{~F} 2^{\prime}, \mathrm{F} 3^{\prime}$, and $\mathrm{F} 3$ were respectively transformed into
Type F2'

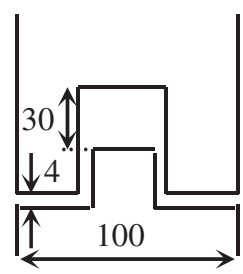

Type F3'

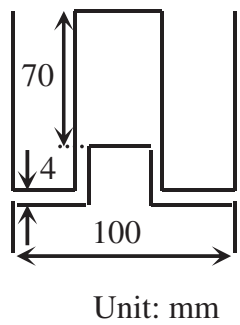

Fig. 10 Cross sections of the bent gaps without absorption treatment.

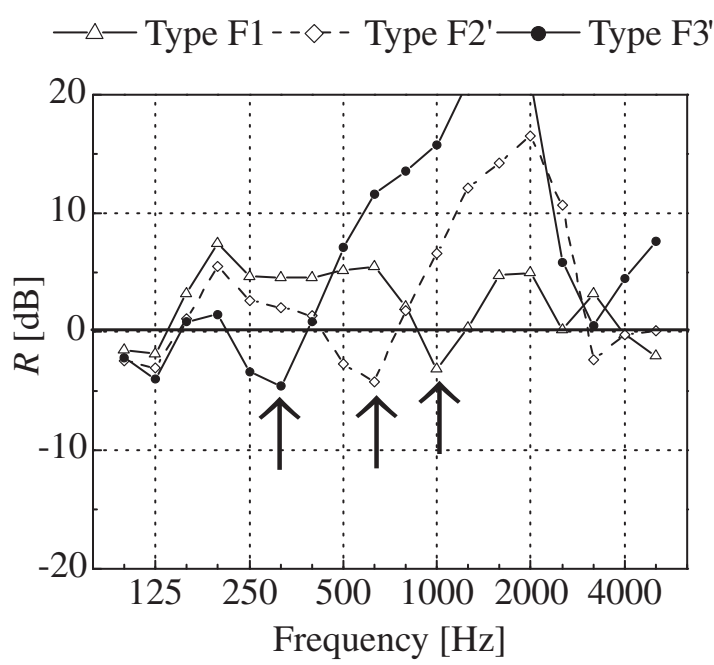

Fig. 11 Measurement results of the bent gaps without absorption treatment.

Types 1, 2, 3, and 3G as one-dimensional sound propagation models shown in Fig. 12. In this transformation, the air spaces were rearranged as muffler devices and the bent parts inside the gaps were rearranged as straight tubes. This rearrangement of the bent parts into straight tubes is considered to have little influence on the transmission characteristics through these parts in the target frequency range. The calculation model for the one-dimensional analysis is shown in Fig. 13. In this figure, progressive and regressive waves at six junctions inside the one-dimensional sound field are respectively indicated as $p_{\text {prog, } i}$ and $p_{\text {reg }, i}(i$ denotes the junction number). The progressive and regressive waves were calculated and the transmission loss $R_{1 \mathrm{D}}$ of this system, which was composed of two tubes and one muffler device, was obtained by

$$
\begin{aligned}
& R_{1 \mathrm{D}}=10 \log \left(\frac{1}{\tau_{\text {reso }}}\right) \\
& \tau_{\text {reso }}=\left|\frac{p_{\text {prog }, 6}}{p_{\text {prog }, 1}}\right|^{2},
\end{aligned}
$$

where $\tau_{\text {reso }}$ is the transmission ratio of the one-dimensional acoustical device. The result of the calculation is shown in 

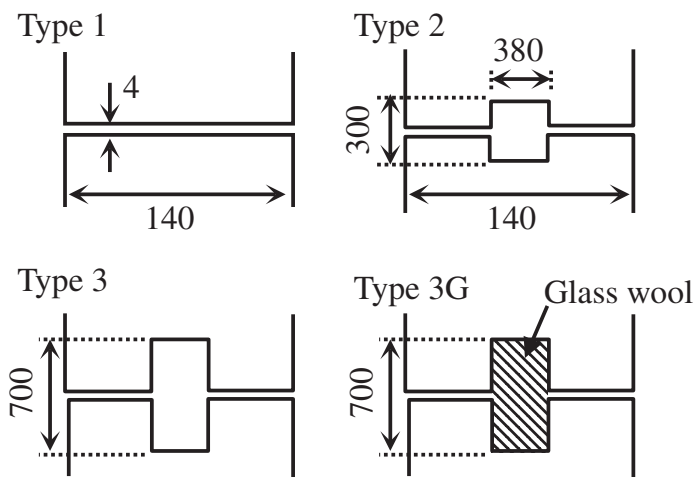

Fig. 12 Cross sections of one-dimensional sound propagation model for bent gaps.

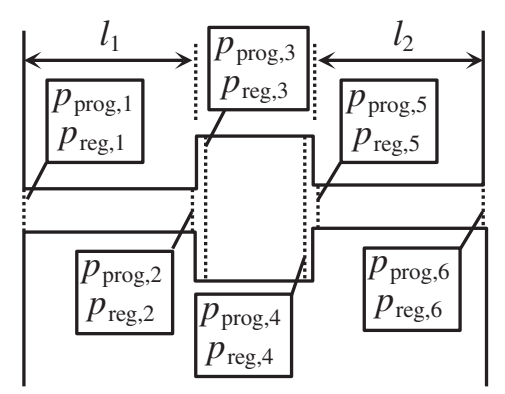

Fig. 13 Calculation model for one-dimensional analysis by the transfer matrix method.

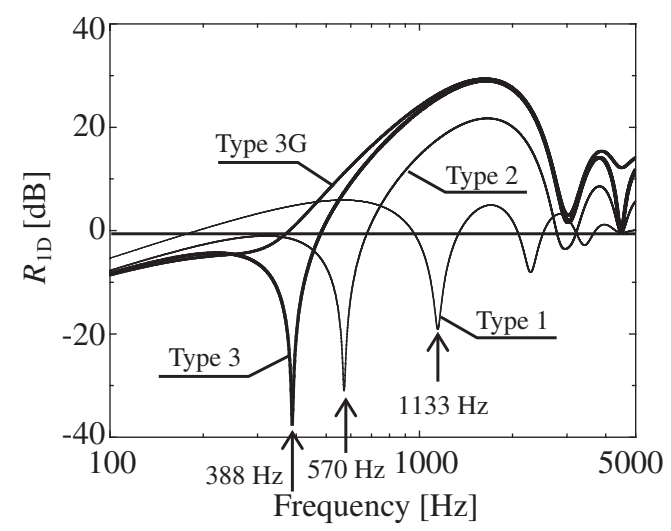

Fig. 14 Calculation results of transmission loss by onedimensional sound propagation models.

Fig. 14. The dip frequencies indicated by arrows decreased as the volume of the air space become larger, and the dip frequencies in Types 1, 2, and 3 agree with those of Types F1, F2', and F3' in Fig. 11, respectively. Compared with Type 3, for Type 3G, the transmission loss in the frequency area around the dip was increased, and this improvement is the same tendency as that in the measurement.

These results indicate that the shift in the dip frequency occurs as a result of the reactive effect of the air space inside the gaps, and as a result of this phenomenon, the

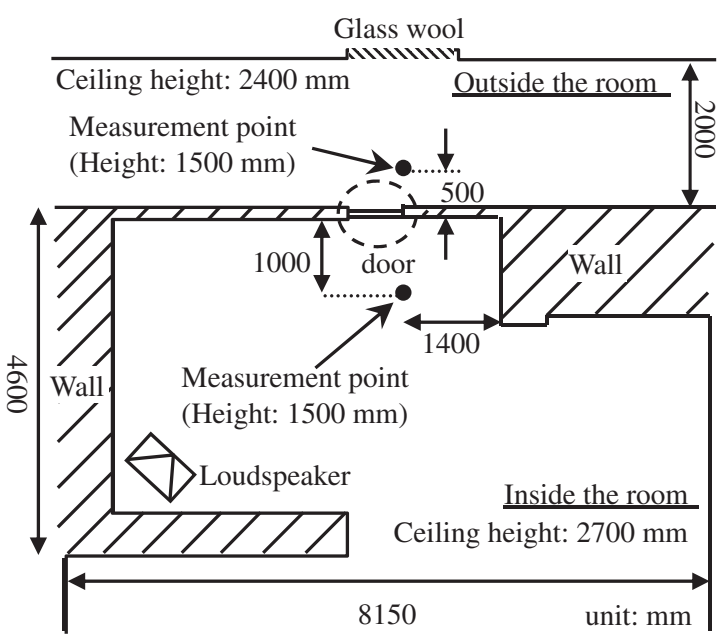

Fig. 15 Plan view of the measurement site.

sound insulation characteristics are deteriorated in lowfrequency ranges. However, the defective sound insulation at the dip frequency can be mitigated by the insertion of the absorption material into the air space.

\section{SOUND TRANSMISSION CHARACTERISTICS OF DOORS WITH GAPS}

In the previous section, the sound transmission loss of gaps with absorption treatment was investigated through numerical and experimental studies. In this section, as a case study, in situ measurement was performed in an office space in order to investigate the effect of absorption treatment inside narrow gaps existing in peripheral parts of a single swing door panel.

\subsection{In Situ Measurement}

Figures 15 and 16 show the plan view of the measurement site, and the elevation and cross sections of the door panel under investigation, respectively. The door panel is made of MDF (medium density fiber board; $700 \mathrm{~kg} / \mathrm{m}^{3}$ density). The dimensions of the door panel are $2,115 \mathrm{~mm}$ $(\mathrm{H}) \times 770 \mathrm{~mm}(\mathrm{~W})$, and absorption treatment with glass wool was applied inside the peripheral gaps on three sides of the door panel, as shown in the cross sections $\mathrm{A}-\mathrm{A}^{\prime}$ and B-B' (Fig. 16). The thickness of the glass wool, $a$, was varied as described below, and the influence on the sound insulation performance was investigated. As shown in Fig. 17, the thickness of the glass wool arranged along the path of the narrow gap was set to three values of 0 (Case 1 , no absorption treatment), 15, and $75 \mathrm{~mm}$ (Cases 2 and 3, respectively). In addition, the measurement was performed for the case where all gaps were filled with clay (Case 0) to check the sound insulation performance of only the panel itself. Then, the width of the air gap was adjusted to $5 \mathrm{~mm}$. 


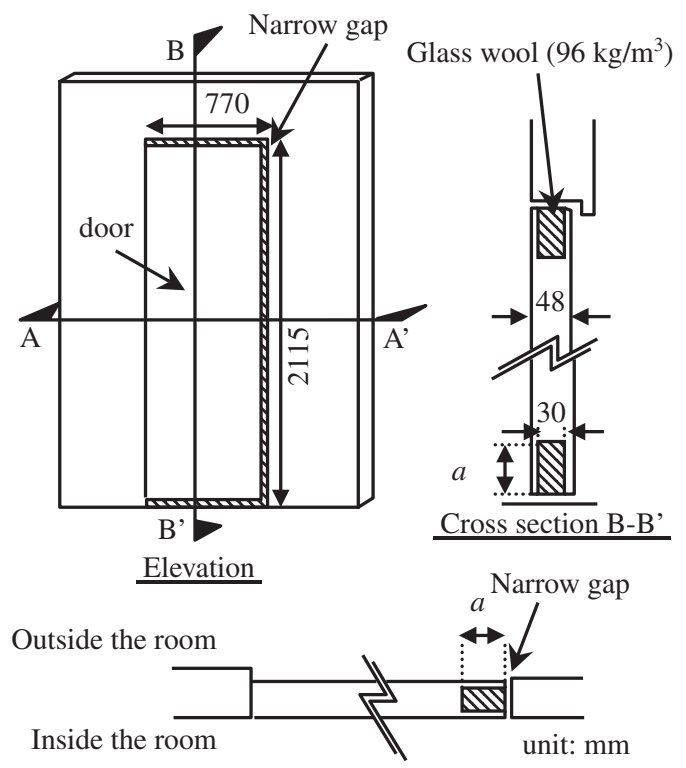

Cross section A-A'

Fig. 16 Elevation and cross sections of the door panel.
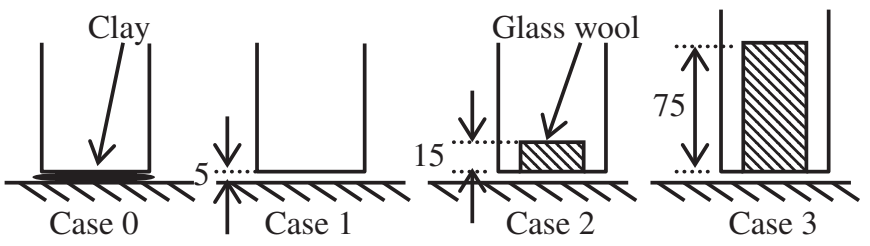

Fig. 17 Treatment of the narrow gap under each measurement condition.

The sound-pressure-level difference between the two points in front of and behind the specimen $D_{p}$ was calculated by

$$
D_{p}=L_{1}-L_{2}
$$

where $L_{1}$ is the sound pressure level measured at the receiving point in the source room and $L_{2}$ is that at the receiving point outside the source room. In order to prevent multiple reflection between the walls, the opposite surface facing the specimen was treated with a glass wool board with dimensions of $900 \mathrm{~mm} \times 1,800 \mathrm{~mm}$, a width of $50 \mathrm{~mm}$, and $32 \mathrm{~kg} / \mathrm{m}^{3}$ density.

\subsection{Numerical Analysis}

The sound insulation of a door is influenced by various complex factors that mainly comprise sound transmission through the peripheral gaps and vibroacoustic transmission through the door panel. For this reason, the sound transmission through the door panel with narrow gaps was simulated by vibroacoustic FDTD analysis. In our past research [19], sound transmission through plaster board was analyzed by the FDTD method and the validity of (a) Cross section A-A'

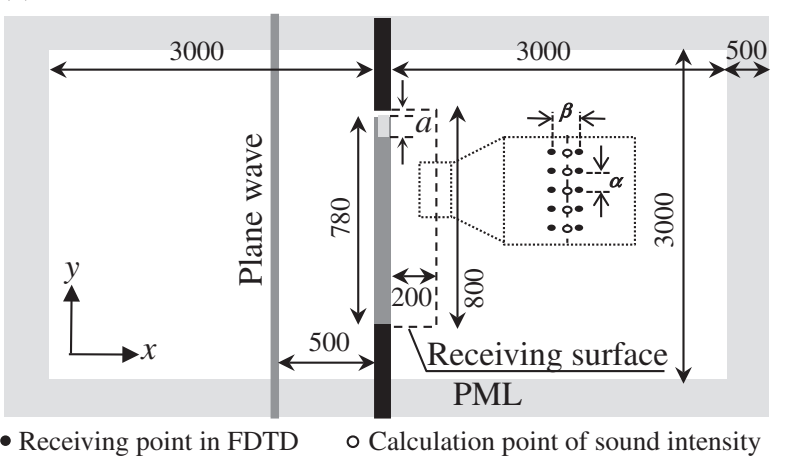

(b) Cross section B-B'

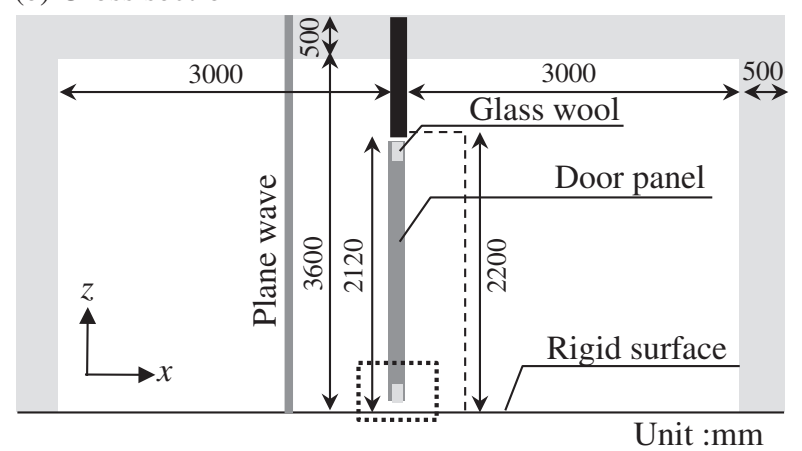

Fig. 18 Cross sections of the one-dimensional sound field for FDTD calculation.

the numerical scheme was confirmed. In this study, the numerical scheme is applied. In this simulation, the sound field is modeled as a three-dimensional field.

The cross sections (a) $\mathrm{A}-\mathrm{A}^{\prime}$ and (b) $\mathrm{B}-\mathrm{B}^{\prime}$ of the door panel are modeled as shown in Fig. 18. In the peripheral parts of the door panel, air gaps and the absorption materials that model the glass wool of $96 \mathrm{~kg} / \mathrm{m}^{3}$ density were set. As the initial condition, a plane-wave sound source was set as shown in the figure, and the sound energy transmitted through the door panel and the peripheral gaps was calculated by the method described in Sect. 2.2. On this occasion, the method of sound incidence into the door panel is different compared with the experimental situation, in which the method of sound incidence shows complex characteristics similar to random incidence. However, it is difficult to model such a complex situation. For this reason, in the simulation, the plane-wave incidence is adopted for simplicity.

To model the shape of the gaps with very narrow width accurately, the FDTD method with a nonuniform-mesh system [20] is applied. For the vibration analysis of the panel, the calculation is performed on the basis of the following bending wave equation which has a term related to the internal damping of a plate.

$$
D \nabla^{4} w+\xi D \frac{\partial}{\partial t} \nabla^{4} w+\mu \frac{\partial^{2} w}{\partial t^{2}}=p
$$




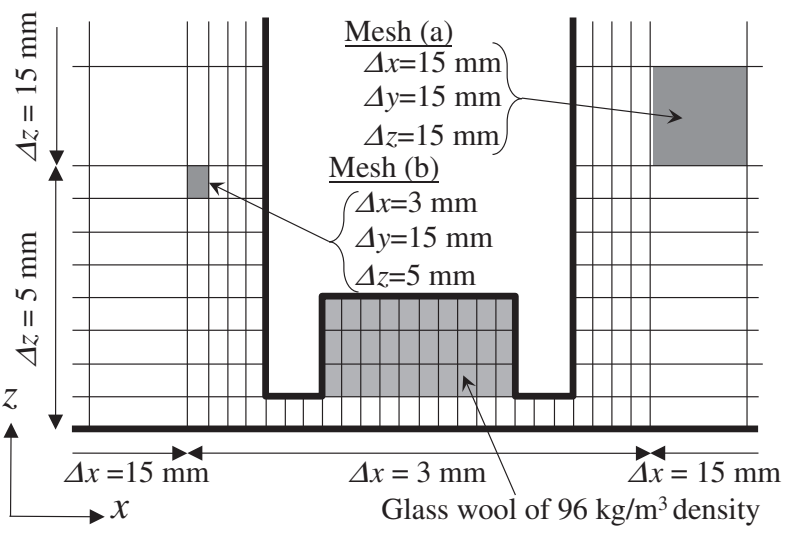

Fig. 19 Detailed mesh structure around the peripheral part of the door panel.

Here, $D$ is the flexural rigidity $\left(D=E h^{3} / 12\left(1-\gamma^{2}\right)\right), E$ is the Young's modulus, $h$ is the thickness of the plate, $\gamma$ is the Poisson's ratio, $\xi$ is the coefficient related to the internal damping, $w$ is the transverse displacement, $\mu$ is the mass density per unit area of the plate, and $p$ is the external force transverse to the plate. The spatial and time differential terms in this equation are approximated by a finite difference, and time development of the bending wave propagation is simulated. The procedure of the coupling analysis between the sound wave and the bending wave is described in [19]. As calculation parameters, $E=2.5 \times$ $10^{9} \mathrm{~N} / \mathrm{m}^{2}, \gamma=0.3, \mu=33.6 \mathrm{~kg} / \mathrm{m}^{2}$, and $\xi=7.96 \times 10^{-4}$ are given for the MDF panel with $48 \mathrm{~mm}$ thickness, and the edge-supporting condition of the MDF plate was set to be simply-supported.

The detailed mesh structure of the sound field around the gap part, surrounded by the broken line in Fig. 18(b), is shown in Fig. 19. In the simulated field, almost all parts of the sound field, except for that around the gap parts, are spatially discretized by (a) a large mesh of $\Delta x=15 \mathrm{~mm}$, $\Delta y=15 \mathrm{~mm}$ and $\Delta z=15 \mathrm{~mm}$. Then, the sound field near the gap is discretized by (b) a small-sized mesh of $\Delta x=$ $3 \mathrm{~mm}, \Delta y=15 \mathrm{~mm}$, and $\Delta z=5 \mathrm{~mm}$, as shown in the figure. As a time interval, $3.125 \times 10^{-3} \mathrm{~ms}$ was set for the fields with both the uniform-mesh and nonuniform-mesh domains.

The normal-incidence absorption coefficient of the surface inside the gap is set to be 0.02 , in the same manner as described in Sect. 2.2. A flow resistance of 50,000 $\mathrm{Ns} / \mathrm{m}^{4}$, is set for glass wool of $96 \mathrm{~kg} / \mathrm{m}^{3}$ density. The porosity is also assumed to be 1.0. The speed of sound and the density inside the material are also set to be the same as those in air. To assume a nonreflection boundary condition, the sound field is terminated with a PML. The normal incidence transmission loss of the door panel with peripheral gaps is calculated using Eq. (8). The sound energy transmitted through the door panel, $L_{J, t}$, is calcu-

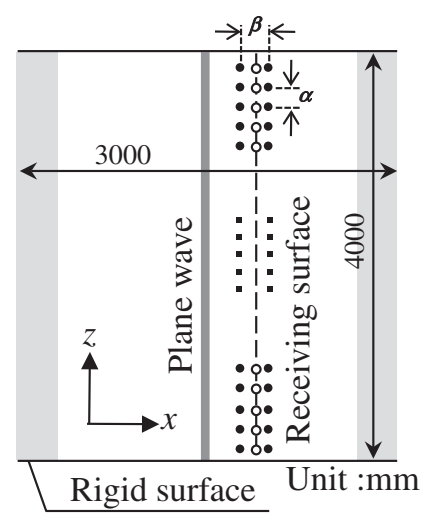

Fig. 20 Cross section of the sound field for calculating the sound energy level of the plane source.

lated by the procedure described in Sect. 2.2. A closed receiving surface of $200 \mathrm{~mm} \times 800 \mathrm{~mm} \times 2,200 \mathrm{~mm}$ is set and, transient responses at a total of 70 receiving points on the surface are used for calculation of the sound intensities. In this calculation, $\alpha$ and $\beta$ are set to be $200 \mathrm{~mm}$ and $50 \mathrm{~mm}$, respectively. The energy of the incident sound to the door panel, $L_{J, i}$, is calculated from the sound energy level of the plane source. The sound energy of the plane source is calculated using the results of another FDTD calculation for the sound field shown in Fig. 20. Two sides of the field are terminated by a PML and the other sides by a rigid boundary. The parameters $\alpha$ and $\beta$ are set to be the same as described above.

\subsection{Results}

Figures 21 and 22 show the measurement and calculation results, respectively. In the calculation results, the transmission loss of Case 0 has almost the same characteristics as that obtained from the mass law. Then, compared with Case 0 , the sound insulation performance in Case 1 is much more deteriorated by the leakage of transmission through peripheral gaps in all frequency bands. In the results of Cases 2 and 3, improvement of the sound insulation is seen in the frequency range between $2 \mathrm{kHz}$ to $5 \mathrm{kHz}$. In the frequency range from $800 \mathrm{~Hz}$ to $1.25 \mathrm{kHz}, R$ is improved. This is considered to be caused by the increase in the thickness of the glass wool.

In the measurement results, the sound insulation performance of Case 1 is also deteriorated compared with that of Case 0 in the frequency range higher than $315 \mathrm{~Hz}$. In the results of Cases 2 and 3, the sound insulation is also improved in the frequency range between $2 \mathrm{kHz}$ to $5 \mathrm{kHz}$. In the frequency range from $315 \mathrm{~Hz}$ to $1.25 \mathrm{kHz}, D_{p}$ is also improved in Case 3.

In order to evaluate the improvement of the sound insulation quantitatively, insertion loss caused by inserting the glass wools into the peripheral gaps was calculated 


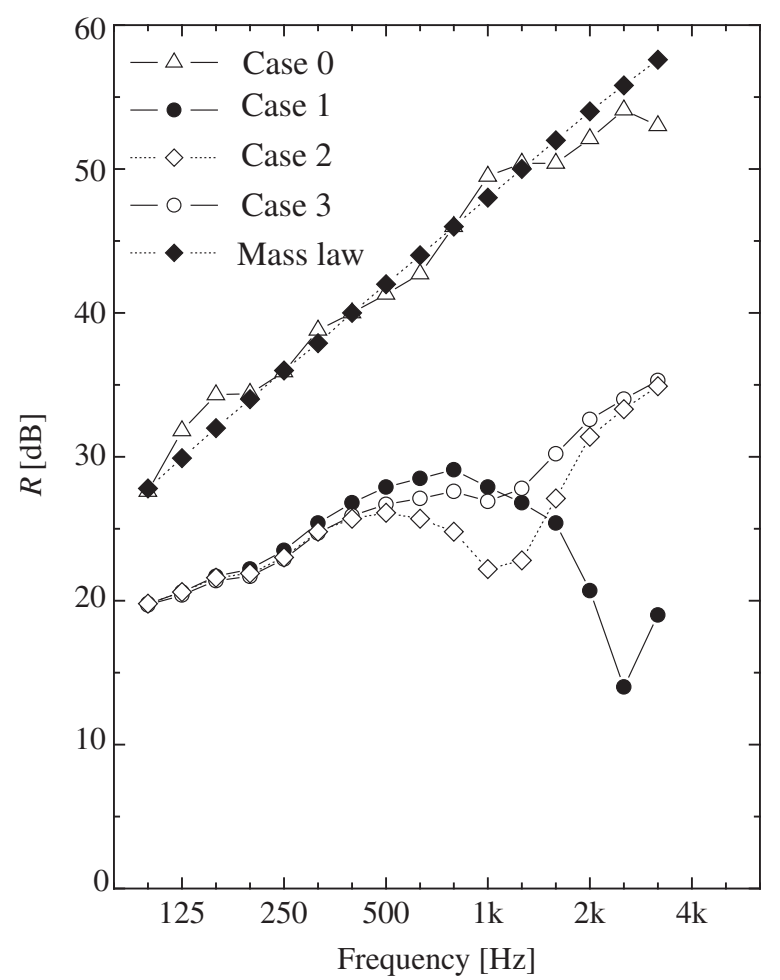

Fig. 21 Calculation results of sound transmission loss of the door panel with peripheral gaps.

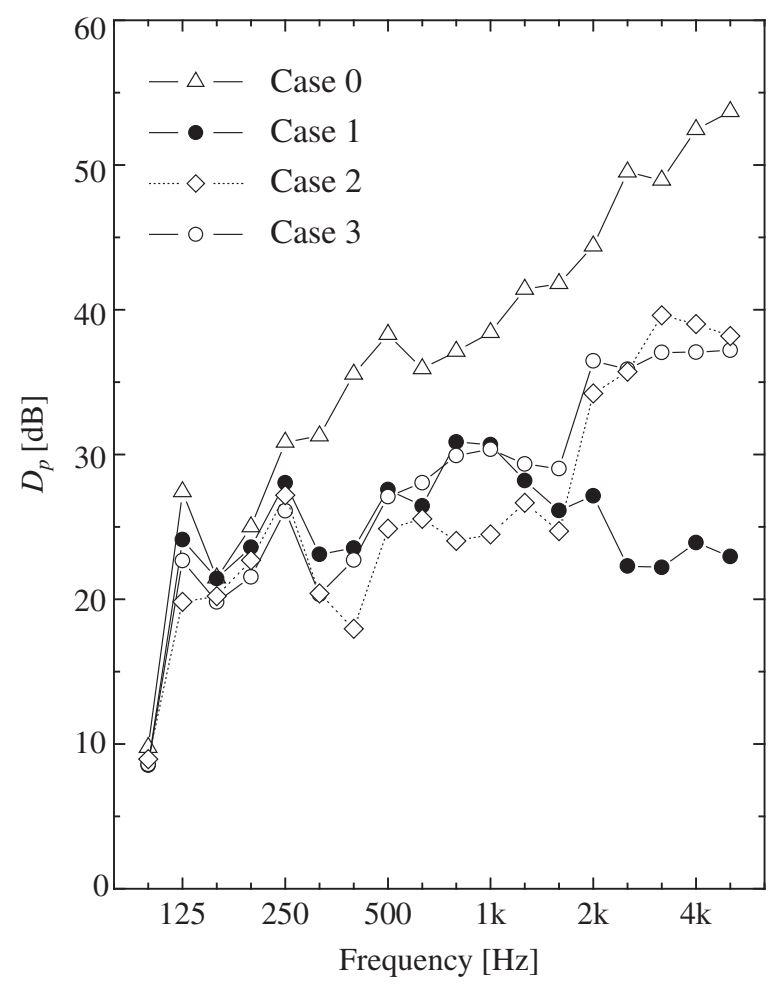

Fig. 22 Measurement results of sound transmission loss of the door panel with peripheral gaps.

using the obtained results. The insertion loss was calculated by subtracting the transmission loss or the sound-pressurelevel difference of Case 1 from that of Case 2 or 3 . The

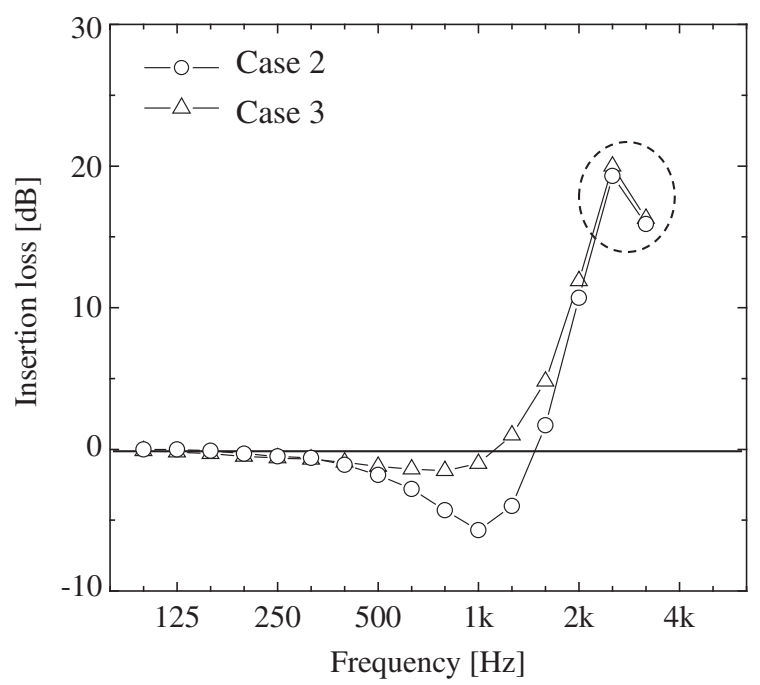

Fig. 23 Insertion loss caused by inserting glass wool inside the peripheral gaps, obtained from FDTD results.

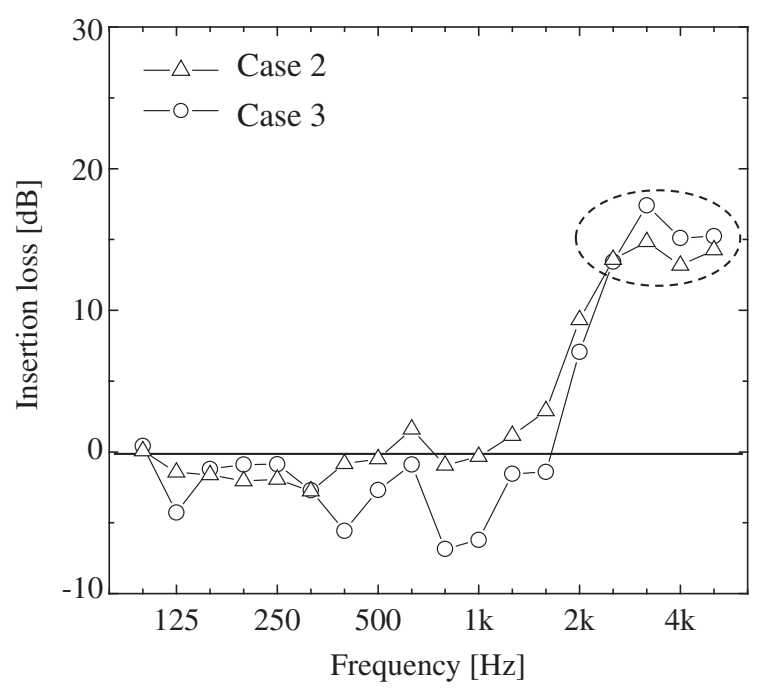

Fig. 24 Insertion loss caused by inserting glass wool inside the peripheral gaps, obtained from measurement results.

results are shown in Figs. 23 and 24. In both figures, insertion loss increases in the frequency range higher than the $2 \mathrm{kHz}$ band, and the insertion loss at frequencies higher than $2.5 \mathrm{kHz}$, as surrounded by a broken line, is between $15 \mathrm{~dB}$ and $20 \mathrm{~dB}$. The numerical result of Case 3 has the highest value of $20 \mathrm{~dB}$ at $2.5 \mathrm{kHz}$, and the measurement result of Case 3 has the highest value of $18 \mathrm{~dB}$ at $3.15 \mathrm{kHz}$. In addition, the following common feature is also seen. The insertion loss decreases to approximately $-6 \mathrm{~dB}$ in the frequency band around $1 \mathrm{kHz}$ in Case 2; however, the deterioration is reduced in Case 3 and the values tend to $0 \mathrm{~dB}$ in both the calculation and measurement results.

These results indicate that the sound insulation characteristics of the door are deteriorated owing to the leakage 
of transmission through the peripheral gaps. However, the leakage of transmission through the gaps can be reduced by inserting an absorption material inside the peripheral gaps.

\section{CONCLUSIONS}

Sound transmission characteristics through narrow gaps with typical shapes modeling those existing in the peripheral parts of doors/windows were investigated through numerical analysis and a full-scale model experiment. As a result, it was found that the leakage of transmission through gaps can be reduced by inserting absorption materials for middle- or high-frequency bands.

As a case study, in situ measurement was performed to investigate the absorption effect caused by adding absorption treatment inside the gaps in the peripheral parts of a door panel. As a result, improvement of the sound insulation of a single swing door by absorption treatment inside the peripheral gaps was confirmed, and it was also indicated that the frequency characteristics of the absorption effect can be predicted by FDTD analysis.

\section{REFERENCES}

[1] F. Ingerslev and A. K. Nilsson, On the Transmission of Sound through Small Apertures and Narrow Slits (G.E.C. Gad, Copenhagen, 1944).

[2] H. Levine and J. Schwinger, "On the theory of diffraction by an aperture in an infinite plane screen," Phys. Rev., 74, 958974 (1948).

[3] Y. Nomura and S. Inawashiro, "On the transmission of acoustic waves through a circular channel of a thick wall," Res. Inst. Electr. Commun. Tohoku Univ., 2, 57-71 (1960).

[4] M. C. Gomperts, "The "sound insulation" of circular and slitshaped aperture," Acustica, 14, 1-16 (1964).

[5] G. P. Wilson and W. W. Soroka, "Approximation to the diffraction of sound by a circular aperture in a rigid wall of finite thickness," J. Acoust. Soc. Am., 37, 286-297 (1965).

[6] P. T. Lewis, "Effect of frame construction on the sound insulation of unsealed windows," Appl. Acoust., 12, 15-24 (1979).

[7] M. A. Burgess, "Resonator effects in window frames," $J$. Sound Vib., 103, 323-332 (1985).

[8] V. Hongisto, "Sound insulation of doors - part 1: Prediction models for structural and leak transmission," J. Sound Vib., 230, 133-148 (2000).

[9] V. Hongisto, J. Keränen and M. Lindgren, "Sound insulation of doors - part 2: Comparison between measurement results and predictions," J. Sound Vib., 230, 149-170 (2000).

[10] T. Iwase, M. Nakajima, T. Ishikawa, Y. Okada and K. Yoshihisa, "Three dimensional observation of sound leakage from slide type window sash using particle velocity sensor," Proc. Inter-Noise 2011 (2011).

[11] T. Asakura, S. Sakamoto, H. Rikitake, Y. Higuchi, F. Satoh and H. Tachibana, "Development of duct-type ventilation system with high sound insulation performance," J. INCE/J, 34, 249-259 (2010) (in Japanese).

[12] H. Suzuki, A. Omoto and K. Fujiwara, "Treatment of boundary conditions by finite difference time domain method," Acoust. Sci. \& Tech., 28, 16-26 (2007).

[13] ISO 15186-1:2000, Acoustics - Measurement of sound insu- lation in buildings and of building elements using sound intensity - Part 1: Laboratory measurements (2000).

[14] H. Kuttruff, Room Acoustics (Elsevier Applied Science, Essex, 2000), pp. 150-161.

[15] T. Yokota, S. Sakamoto and H. Tachibana, "Visualization of sound propagation and scattering in rooms," Acoust. Sci. \& Tech., 23, 40-46 (2002).

[16] J. P. Berenger, "A perfectly matched layer for the absorption of electromagnetic waves," J. Comput. Phys., 114, 185-200 (1994).

[17] H. Tachibana and H. Yano, Measurement Methods for Environmental Noise and Building Acoustics (Corona Publishing, Tokyo, 2004), pp. 161-162 (in Japanese).

[18] H. Nakagawa, S. Ohashi, Y. Ohara, T. Ogawa, H. Shima and M. Yamaguchi, "Acoustic characteristic of a wide variety of porous materials," Trans. Tech. Comm. Archit. Acoust. Acoust. Soc. Jpn., AA94-27 (1994) (in Japanese).

[19] T. Asakura and S. Sakamoto, "Finite-difference time-domain analysis of sound insulation performance of wall systems," Build. Acoust., 16, 267-281 (2009).

[20] T. Asakura and S. Sakamoto, "Finite-difference time-domain analysis on leak transmission characteristics of narrow gaps," Acoust. Sci. \& Tech., 32, 182-193 (2011).

[21] M. E. Delany and E. N. Bazley, "Acoustical properties of fibrous absorbent materials,” Appl. Acoust., 3, 105-116 (1970).

\section{Appendix}

The calculation method for the one-dimensional sound propagation model applied in this research is described in this appendix. The one-dimensional model comprising one muffler and two extension tubes as shown in Fig. 13, is considered. In this system, six junctions are set as shown in the figure and 12 parameters of complex amplitudes for the progressive and regressive waves at all junctions are ultimately obtained from this calculation.

Firstly, the relationships between the parameters at junction 1 versus 2, junction 3 versus 4 , and junction 5 versus 6 are described as

$$
\begin{aligned}
\left(\begin{array}{c}
p_{\text {reg }, 1} \\
p_{\text {prog }, 2}
\end{array}\right) & =\left(\begin{array}{cc}
0 & e^{-j k l_{1}} \\
e^{-j k l_{1}} & 0
\end{array}\right)\left(\begin{array}{c}
p_{\text {prog }, 1} \\
p_{\text {reg }, 2}
\end{array}\right) \\
\left(\begin{array}{c}
p_{\text {reg }, 3} \\
p_{\text {prog }, 4}
\end{array}\right) & =\left(\begin{array}{cc}
0 & e^{-j k l_{\mathrm{m}}} \\
e^{-j k l_{\mathrm{m}}} & 0
\end{array}\right)\left(\begin{array}{c}
p_{\text {prog }, 3} \\
p_{\text {reg }, 4}
\end{array}\right) \\
\left(\begin{array}{c}
p_{\text {reg }, 5} \\
p_{\text {prog }, 6}
\end{array}\right) & =\left(\begin{array}{cc}
0 & e^{-j k l_{2}} \\
e^{-j k l_{2}} & 0
\end{array}\right)\left(\begin{array}{c}
p_{\text {prog, }, 5} \\
p_{\text {reg }, 6}
\end{array}\right),
\end{aligned}
$$

where $k$ is the wave number and $l_{1}$ and $l_{2}$ are the lengths of the extension tubes, and $l_{\mathrm{m}}$ is the length of the muffler. For the situation that an absorption material is inserted into the propagation path, the damping of the sound traveling in the material is modeled by using the complexvalued propagation constant obtained from the DelanyBazley model [21].

Secondly, the relationships between the parameters of junction 2 versus 3 and junction 4 versus 5 are described as 


$$
\begin{aligned}
\left(\begin{array}{l}
p_{\text {reg, }, 2} \\
p_{\text {prog, } 3}
\end{array}\right) & =\frac{1}{1+m}\left(\begin{array}{cc}
1-m & 2 m \\
2 & m-1
\end{array}\right)\left(\begin{array}{c}
p_{\text {prog, } 2} \\
p_{\text {reg, } 3}
\end{array}\right) \quad(\mathrm{A} \cdot 4) \\
\left(\begin{array}{l}
p_{\text {reg, }, 4} \\
p_{\text {prog }, 5}
\end{array}\right) & =\frac{1}{1+m^{-1}}\left(\begin{array}{cc}
1-m^{-1} & 2 m^{-1} \\
2 & m^{-1}-1
\end{array}\right)\left(\begin{array}{c}
p_{\text {prog, }, 4} \\
p_{\text {reg }, 5}
\end{array}\right)
\end{aligned}
$$

$$
m=\frac{S_{\mathrm{muf}}}{S_{\mathrm{ext}}},
$$

where $S_{\text {muf }}$ and $S_{\text {ext }}$ are areas of the cross sections of the muffler and the extension tubes, respectively. Equations (A.4) and (A.5) describe the reactive effect of sound caused by expansion and contraction of the path, respectively.

Thirdly, the reflections of the sound wave at the terminal boundaries at junctions 1 and 6 are described as

$$
\begin{aligned}
& p_{\text {prog }, 1}=g_{1}+T_{1} p_{\text {reg }, 1} \\
& p_{\text {reg, } 6}=T_{6} p_{\text {prog }, 6}
\end{aligned}
$$

$$
\begin{aligned}
& T_{1}=\left|T_{1}\right| e^{-2 j k \Delta l_{\mathrm{M}}} \\
& T_{6}=\left|T_{6}\right| e^{-2 j k \Delta l_{\mathrm{M}}},
\end{aligned}
$$

where $T_{1}$ and $T_{6}$ are complex reflection factors at the terminal boundaries, $g_{1}$ is the sound wave input to the system, and $\Delta l_{\mathrm{M}}$ is the correction length of the opening. In this case, the width of the slit is $4 \mathrm{~mm}$ which is relatively small compared with the wavelength of the target frequency range (up to $5 \mathrm{kHz}$ ). For this reason, the absolute values of the reflection constants, $\left|T_{1}\right|$ and $\left|T_{6}\right|$ are assumed to be 1.0. The correction length is estimated as below, equation, referring to the paper by Gomperts [4].

$$
\Delta l_{\mathrm{M}}=\sqrt{\frac{\left(\ln \frac{k a}{8}+0.577\right)^{2}+\left(\frac{\pi}{2}\right)^{2}}{\pi^{2}}} \cdot a
$$

Here, $a$ is the width of the tube. Finally, Eqs. (A.1), (A.2), $(\mathrm{A} \cdot 3),(\mathrm{A} \cdot 4),(\mathrm{A} \cdot 5),(\mathrm{A} \cdot 7)$, and $(\mathrm{A} \cdot 8)$ are solved together and 12 parameters for every frequency are obtained. 